Kragujevac Journal of Mathematics

Volume 38(1) (2014), Pages 195-202.

\title{
ON SOME SEQUENCE SPACES OF NON-ABSOLUTE TYPE
}

\author{
SINAN ERCAN ${ }^{1}$ AND CIĞDEM A. BEKTAŞ ${ }^{2}$
}

\begin{abstract}
In this paper, we introduce the notion of $\lambda_{v}$-convergent and bounded sequences. Further, we introduce the spaces $\ell_{\infty}^{\lambda}\left(\triangle_{v}\right), c_{0}^{\lambda}\left(\triangle_{v}\right)$ and $c^{\lambda}\left(\triangle_{v}\right)$, which are BK-spaces of non-absolute type and we prove that these spaces are linearly isomorphic to the spaces $\ell_{\infty}, c_{0}$ and $c$, respectively. Moreover, we establish some inclusion relations between these spaces.
\end{abstract}

\section{INTRODUCTION}

A sequence space is defined to be a linear space of real or complex sequences. Let $w$ denote the spaces of all complex sequences. If $x \in w$, then we simply write $x=\left(x_{k}\right)$ instead of $x=\left(x_{k}\right)_{n=0}^{\infty}$.

Let $X$ be a sequence space. If $X$ is a Banach space and

$$
\tau_{k}: X \rightarrow C, \tau_{k}(x)=x_{k} \quad(k=1,2, \ldots)
$$

is a continuous for all $k, X$ is called a BK-space.

We shall write $\ell_{\infty}, c$ and $c_{0}$ for the sequence spaces of all bounded, convergent and null sequences, respectively, which are BK-spaces with the same norm given by

$$
\|x\|_{\infty}=\sup _{k}\left|x_{k}\right|
$$

for all $k \in N$.

M. Mursaleen and A. K. Noman [10] introduced the sequence spaces $\ell_{\infty}^{\lambda}, c^{\lambda}$ and $c_{0}^{\lambda}$ as the sets of all $\lambda$-bounded, $\lambda$-convergent and $\lambda$-null sequences, respectively, that is

$$
\ell_{\infty}^{\lambda}=\left\{x \in w: \sup _{n}\left|\Lambda_{n}(x)\right|<\infty\right\}
$$

Key words and phrases. Sequence spaces of non-absolute type, BK-spaces, Difference Sequence Spaces.

2010 Mathematics Subject Classification. Primary: 46A45. Secondary: 46B20.

Received: March 5, 2014

Revised: April 10, 2014. 


$$
\begin{aligned}
& c^{\lambda}=\left\{x \in w: \lim _{n \rightarrow \infty} \Lambda_{n}(x) \text { exists }\right\}, \\
& c_{0}^{\lambda}=\left\{x \in w: \lim _{n \rightarrow \infty} \Lambda_{n}(x)=0\right\},
\end{aligned}
$$

where $\Lambda_{n}(x)=\frac{1}{\lambda_{n}} \sum_{k=0}^{n}\left(\lambda_{k}-\lambda_{k-1}\right) x_{k}, k \in \mathbb{N}$.

H. Ganie and N. A. Sheikh [7] introduced the spaces $c_{0}\left(\triangle_{u}^{\lambda}\right)$ and $c\left(\triangle_{u}^{\lambda}\right)$ as follows:

$$
\begin{aligned}
c\left(\Delta_{u}^{\lambda}\right) & =\left\{x \in w: \lim _{n \rightarrow \infty} \hat{\Lambda}_{n}(x) \text { exists }\right\}, \\
c_{0}\left(\Delta_{u}^{u}\right) & =\left\{x \in w: \lim _{n \rightarrow \infty} \hat{\Lambda}_{n}(x)=0\right\},
\end{aligned}
$$

where $\hat{\Lambda}_{n}(x)=\frac{1}{\lambda_{n}} \sum_{k=0}^{n}\left(\lambda_{k}-\lambda_{k-1}\right) u_{k}\left(x_{k}-x_{k-1}\right), \quad k \in \mathbb{N}$.

Let $v=\left(v_{k}\right)$ be any fixed sequence of non-zero complex numbers. Colak [6] defined the sequence spaces $\ell_{\infty}\left(\Delta_{v}\right), c\left(\Delta_{v}\right)$ and $c_{0}\left(\Delta_{v}\right)$ as follows:

$$
\begin{aligned}
\ell_{\infty}\left(\Delta_{v}\right) & =\left\{x \in w: \Delta_{v} x_{k} \in \ell_{\infty}\right\}, \\
c\left(\triangle_{v}\right) & =\left\{x \in w: \Delta_{v} x_{k} \in c\right\}, \\
c_{0}\left(\triangle_{v}\right) & =\left\{x \in w: \Delta_{v} x_{k} \in c_{0}\right\},
\end{aligned}
$$

where $\Delta_{v} x_{k}=v_{k} x_{k}-v_{k-1} x_{k-1}$.

Several authors have recently introduced new sequence spaces, see for instance $[1,2,11]$.

\section{Notion of $\lambda_{v}$-CONVERGENT AND BOUNDED SEQUENCES}

Throughout this paper, let $\lambda=\left(\lambda_{k}\right)$ be a strictly increasing sequence of positive reals tending to infinity, $0<\lambda_{0}<\lambda_{1}<\ldots$ and $\lambda_{k} \rightarrow \infty$ as $k \rightarrow \infty$. We take

$$
\tilde{\wedge}_{n}(x)=\frac{1}{\lambda_{n}} \sum_{k=0}^{n}\left(\lambda_{k}-\lambda_{k-1}\right) \triangle_{v} x_{k}
$$

where $\Delta_{v} x_{k}=v_{k} x_{k}-v_{k-1} x_{k-1}$.

A sequence $x=\left(x_{k}\right) \in w$ is $\lambda_{v}$-convergent to the number $\ell \in C$, called as the $\lambda_{v^{-}}$ limit of $x$, if $\tilde{\Lambda}_{n}(x) \rightarrow \ell$ as $n \rightarrow \infty$. In particular, we say that $x$ is a $\lambda_{v}$-null sequence if $\tilde{\Lambda}_{n}(x) \rightarrow 0$ as $n \rightarrow \infty$. Further we say that $x$ is $\lambda_{v}$-bounded if $\sup \left|\tilde{\Lambda}_{n}(x)\right|<\infty$. Negative subscript is equal to naught. For instance, $\lambda_{-1}=0$ and $x_{-1}^{n}=0$. We have

$$
\lim _{n \rightarrow \infty}\left|\tilde{\wedge}_{n}(x)-a\right|=\lim _{n \rightarrow \infty}\left|\frac{1}{\lambda_{n}} \sum_{k=0}^{n}\left(\lambda_{k}-\lambda_{k-1}\right)\left(\Delta_{v} x_{k}-a\right)\right|=0 .
$$

So we can say by (2.2) $\lim _{n \rightarrow \infty} \tilde{\wedge}_{n}(x)=a$. Hence $x$ is $\lambda_{v}$-convergent to $a$.

Lemma 2.1. Every convergent sequence is $\lambda_{v}$-convergent to the same ordinary limit. 
The following result is immediate by Lemma 2.1 .

Lemma 2.2. If a $\lambda_{v}$-convergent sequence converges in the ordinary sense, then it must converge to the same $\lambda_{v}$-limit.

Let $x=\left(x_{k}\right) \in w$ and $n \geq 1$. Then by using (2.1) we derive that

$$
\begin{aligned}
\Delta_{v} x_{n}-\tilde{\Lambda}_{n}(x) & =\Delta_{v} x_{n}-\frac{1}{\lambda_{n}} \sum_{k=0}^{n}\left(\lambda_{k}-\lambda_{k-1}\right) \Delta_{v} x_{k} \\
& =\frac{1}{\lambda_{n}} \sum_{k=0}^{n-1}\left(\lambda_{k}-\lambda_{k-1}\right) \sum_{i=k+1}^{n}\left(\Delta_{v} x_{i}-\Delta_{v} x_{i-1}\right) \\
& =\frac{1}{\lambda_{n}} \sum_{i=1}^{n}\left(\Delta_{v} x_{i}-\Delta_{v} x_{i-1}\right) \sum_{k=0}^{i-1}\left(\lambda_{k}-\lambda_{k-1}\right) \\
& =\frac{1}{\lambda_{n}} \sum_{i=1}^{n} \lambda_{i-1}\left(\Delta_{v} x_{i}-\Delta_{v} x_{i-1}\right)
\end{aligned}
$$

Therefore we have for every $x=\left(x_{k}\right) \in w$ that

$$
\Delta_{v} x_{n}-\tilde{\Lambda}_{n}(x)=S_{n}(x) \quad(n \in \mathbb{N})
$$

where the sequence $S(x)=\left(S_{n}(x)\right)_{n=0}^{\infty}$ is defined by

$$
S_{0}(x)=0 \quad \text { and } \quad S_{n}(x)=\frac{1}{\lambda_{n}} \sum_{i=1}^{n} \lambda_{i-1}\left(\Delta_{v} x_{i}-\Delta_{v} x_{i-1}\right), \quad(n \geq 1) .
$$

Lemma 2.3. A $\lambda_{v}$-convergent sequence $x$ converges in the ordinary sense if and only if $S(x) \in c_{0}$.

Proof. Let $x=\left(x_{n}\right)$ be $\lambda_{v}$-convergent sequence in the ordinary sense. Then, from Lemma 2.2 we have $x=\left(x_{n}\right)$ converges to the same $\lambda_{v}$-limit. We obtain $S(x) \in c_{0}$ by (2.3). Conversely, let $S(x) \in c_{0}$. We have

$$
\lim _{n \rightarrow \infty} \Delta_{v} x_{n}=\lim _{n \rightarrow \infty} \tilde{\wedge}_{n}(x) .
$$

From the above equation, we deduce that $\lambda_{v}$-convergent sequence $x$ converges in the ordinary sense.

Lemma 2.4. Every bounded sequence is $\lambda_{v}$-bounded.

Lemma 2.5. A $\lambda_{v}$-bounded sequence $x$ is bounded in the ordinary sense if and only if $S(x) \in \ell_{\infty}$.

Proof. We can obtain it directly from Lemma 2.4 and (2.4). 


\section{The SPACES of $\lambda_{v}$-CONVERGENT AND Bounded SEQUenCES}

In this section, we introduce the sequence space $\ell_{\infty}^{\lambda}\left(\Delta_{v}\right), c^{\lambda}\left(\Delta_{v}\right)$ and $c_{0}^{\lambda}\left(\Delta_{v}\right)$ as the sets of all $\lambda_{v}$-bounded, $\lambda_{v}$-convergent and $\lambda_{v}$-null sequences;

$$
\begin{aligned}
\ell_{\infty}^{\lambda}\left(\Delta_{v}\right) & =\left\{x \in w: \sup \left|\tilde{\Lambda}_{n}(x)\right|<\infty\right\}, \\
c^{\lambda}\left(\Delta_{v}\right) & =\left\{x \in w: \lim _{n \rightarrow \infty} \tilde{\Lambda}_{n}(x) \text { exists }\right\} \\
c_{0}^{\lambda}\left(\Delta_{v}\right) & =\left\{x \in w: \lim _{n \rightarrow \infty} \tilde{\Lambda}_{n}(x)=0\right\}
\end{aligned}
$$

where $\tilde{\Lambda}_{n}(x)$ like $(2.1)$.

Theorem 3.1. The sequence spaces $\ell_{\infty}^{\lambda}\left(\Delta_{v}\right), c^{\lambda}\left(\Delta_{v}\right)$ and $c_{0}^{\lambda}\left(\Delta_{v}\right)$ are BK-spaces with the same norm given by

$$
\|x\|_{\ell_{\infty}^{\lambda}\left(\Delta_{v}\right)}=\left\|\tilde{\Lambda}_{n}(x)\right\|_{\ell_{\infty}}=\sup _{n}\left|\tilde{\Lambda}_{n}(x)\right| .
$$

Proof. The proof is seen eaisly. So it is omitted.

Remark 3.1. We can see that the absolute property does not hold on the spaces $\ell_{\infty}^{\lambda}\left(\Delta_{v}\right), c^{\lambda}\left(\Delta_{v}\right)$ and $c_{0}^{\lambda}\left(\Delta_{v}\right)$. For at least one sequence $x$ in each of these spaces have that $\|x\|_{\ell_{\infty}^{\lambda}\left(\Delta_{v}\right)} \neq\||x|\|_{\ell_{\infty}^{\lambda}\left(\Delta_{v}\right)}$, where $|x|=\left(\left|x_{k}\right|\right)$. So these spaces are BK-spaces of non-absolute type.

Theorem 3.2. The sequence spaces $\ell_{\infty}^{\lambda}\left(\Delta_{v}\right), c^{\lambda}\left(\Delta_{v}\right)$ and $c_{0}^{\lambda}\left(\Delta_{v}\right)$ are linearly isomorphic to the spaces $\ell_{\infty}, c$ and $c_{0}$.

Proof. We only consider the case $c_{0}^{\lambda}\left(\Delta_{v}\right) \cong c_{0}$. The cases $c^{\lambda}\left(\Delta_{v}\right) \cong c$ and $\ell_{\infty}^{\lambda}\left(\Delta_{v}\right) \cong$ $\ell_{\infty}$ can be shown similarly. To prove the theorem, we must show the existence of linear bijection between $c_{0}^{\lambda}\left(\Delta_{v}\right)$ and $c_{0}$. Consider the transformation $T$ defined, $T x=\tilde{\Lambda}(x) \in c_{0}$ for every $x \in c_{0}^{\lambda}\left(\Delta_{v}\right)$. The linearity of $T$ is obvious. It is trivial that $x=0$ whenever $T x=0$ and hence $T$ is injective.

To show surjective we define the sequence $x=\left\{x_{k}(\lambda)\right\}$ by

$$
x_{k}(\lambda)=v_{k}^{-1} \sum_{j=0}^{k} \sum_{i=j-1}^{j}(-1)^{j-i} \frac{\lambda_{i}}{\left(\lambda_{j}-\lambda_{j-1}\right)} y_{i} \quad \text { for } \quad k \in \mathbb{N} .
$$

We have that

$$
\tilde{\Lambda}_{n}(x)=\frac{1}{\lambda_{n}} \sum_{k=0}^{n} \sum_{i=k-1}^{k}(-1)^{k-i} \lambda_{i} y_{i}=y_{n} .
$$

We can say that $\tilde{\Lambda}_{n}(x)=y_{n}$ from (3.1) and $y \in c_{0}$, hence $\tilde{\Lambda}_{n}(x) \in c_{0}$. We deduce from that $x \in c_{0}^{\lambda}\left(\triangle_{v}\right)$ and $T x=y$. Hence $T$ is surjective.

We have for every $x \in c_{0}^{\lambda}\left(\triangle_{v}\right)$ that

$$
\|T x\|_{c_{0}}=\|T x\|_{\ell_{\infty}}=\|\tilde{\Lambda}(x)\|_{\ell_{\infty}}=\|x\|_{c_{0}^{\lambda}\left(\triangle_{v}\right)}
$$


which means that $c_{0}^{\lambda}\left(\triangle_{v}\right)$ and $c_{0}$ are linearly isomorphic. Similarly we can obtain that $c^{\lambda}\left(\Delta_{v}\right) \cong c$ and $\ell_{\infty}^{\lambda}\left(\Delta_{v}\right) \cong \ell_{\infty}$.

\section{Some Inclusion Relations}

Theorem 4.1. The inclusions $c_{0}^{\lambda}\left(\Delta_{v}\right) \subset c^{\lambda}\left(\Delta_{v}\right) \subset \ell_{\infty}^{\lambda}\left(\Delta_{v}\right)$ strictly hold.

Proof. It is obvious that the inclusions $c_{0}^{\lambda}\left(\Delta_{v}\right) \subset c^{\lambda}\left(\Delta_{v}\right) \subset \ell_{\infty}^{\lambda}\left(\Delta_{v}\right)$ hold. Furthermore, since the inclusion $c_{0} \subset c$ is strict, it follows by Lemma 2.1 that the inclusion $c_{0}^{\lambda}\left(\Delta_{v}\right) \subset c^{\lambda}\left(\Delta_{v}\right)$ is also strict. Consider the sequence $x=\left(x_{k}\right)$ defined by

$$
x_{k}=v_{k}^{-1} \sum_{i=1}^{k}(-1)^{i}\left(\lambda_{i}+\lambda_{i-1}\right) /\left(\lambda_{i}-\lambda_{i-1}\right)
$$

for all $k \in \mathbb{N}$. We obtain

$$
\Delta_{v} x_{k}=(-1)^{k}\left(\lambda_{k}+\lambda_{k-1}\right) /\left(\lambda_{k}+\lambda_{k-1}\right)
$$

for $k \in \mathbb{N}$. Then we have for every $n \in \mathbb{N}$ that

$$
\tilde{\Lambda}_{n}(x)=\frac{1}{\lambda_{n}} \sum_{k=0}^{n}(-1)^{k}\left(\lambda_{k}+\lambda_{k-1}\right)=(-1)^{n} .
$$

We can say that $\tilde{\Lambda}_{n}(x) \in \ell_{\infty} / c$. Hence the sequence $x$ is in $\ell_{\infty}^{\lambda}\left(\Delta_{v}\right)$ but not in $c^{\lambda}\left(\Delta_{v}\right)$. So the inclusion $c^{\lambda}\left(\Delta_{v}\right) \subset \ell_{\infty}^{\lambda}\left(\Delta_{v}\right)$ strictly holds. This completes proof.

Theorem 4.2. The inclusion $\ell_{\infty}\left(\Delta_{v}\right) \subset \ell_{\infty}^{\lambda}\left(\Delta_{v}\right)$ holds.

Proof. Let $x \in \ell_{\infty}\left(\Delta_{v}\right)$. Then we deduce that

$$
\frac{1}{\lambda_{n}} \sum_{k=0}^{\infty}\left(\lambda_{k}-\lambda_{k-1}\right)\left|\Delta_{v} x_{k}\right| \leq \frac{1}{\lambda_{n}} \sup _{k}\left|\Delta_{v} x_{k}\right| \sum_{k=0}^{n}\left(\lambda_{k}-\lambda_{k-1}\right)=\sup _{k}\left|\Delta_{v} x_{k}\right|<\infty .
$$

Hence, $x \in \ell_{\infty}^{\lambda}\left(\Delta_{v}\right)$.

The following result is immediate by the regularity of the matrix $\tilde{\Lambda}$ and by Lemma 2.3 .

Lemma 4.1. The inclusions $c_{0} \subset c_{0}^{\lambda}\left(\Delta_{v}\right)$ and $c \subset c^{\lambda}\left(\Delta_{v}\right)$ hold. Furthermore, the equalities hold if and only if $S(x) \in c_{0}$ for every sequence $x$ in the spaces $c_{0}^{\lambda}\left(\Delta_{v}\right)$ and $c^{\lambda}\left(\Delta_{v}\right)$, respectively.

Proof. $c_{0} \subset c_{0}^{\lambda}\left(\Delta_{v}\right)$ and $c \subset c^{\lambda}\left(\Delta_{v}\right)$ are obvious from Lemma 2.1. To prove second part we suppose firstly equality $c=c_{0}^{\lambda}\left(\Delta_{v}\right)$ holds. Then, we have for every $x \in c_{0}^{\lambda}\left(\Delta_{v}\right)$ that $x \in c_{0}$ and hence $S(x) \in c_{0}$ by Lemma 2.3. Conversely, let $x \in c_{0}^{\lambda}\left(\Delta_{v}\right)$. Then, we have that $S(x) \in c_{0}$. Thus, it follows by Lemma 2.3 and then Lemma 2.2, that $x \in c_{0}$. This shows that the inclusion $c_{0}^{\lambda}\left(\Delta_{v}\right) \subset c_{0}$ holds. Hence by combining the inclusions $c_{0}^{\lambda}\left(\Delta_{v}\right) \subset c_{0}$ and $c_{0} \subset c_{0}^{\lambda}\left(\Delta_{v}\right)$, we get the equality $c_{0}^{\lambda}\left(\Delta_{v}\right)=c_{0}$.

We can similarly show the equality $c=c^{\lambda}\left(\Delta_{v}\right)$ holds if and only if $S(x) \in c_{0}$ for every $x \in c^{\lambda}\left(\Delta_{v}\right)$. 
Lemma 4.2. The inclusion $\ell_{\infty} \subset \ell_{\infty}^{\lambda}\left(\Delta_{v}\right)$ holds. Furthermore, the equality $\ell_{\infty}=$ $\ell_{\infty}^{\lambda}\left(\Delta_{v}\right)$ holds if and only if $S(x) \in \ell_{\infty}$ for every $x \in \ell_{\infty}^{\lambda}\left(\Delta_{v}\right)$.

It can be seen clearly from by Lemma 4.1 we can say that $c_{0} \subset c_{0}^{\lambda}\left(\Delta_{v}\right) \cap c$. Conversely, it follows by Lemma 2.2 that $c_{0}^{\lambda}\left(\Delta_{v}\right) \cap c \subset c_{0}$. Hence the following result can be derived.

Theorem 4.3. The equality $c_{0}^{\lambda}\left(\Delta_{v}\right) \cap c=c_{0}$ holds.

Let $x=\left(x_{k}\right) \in w$ and $n \geqslant 1$. Then, from (2.3) and (2.4), we derive that

$$
\begin{aligned}
S_{n}(x) & =\frac{1}{\lambda_{n}} \sum_{k=1}^{n} \lambda_{k-1}\left(\Delta_{v} x_{k}-\Delta_{v} x_{k-1}\right) \\
& =\frac{1}{\lambda_{n}}\left[\sum_{k=1}^{n} \lambda_{k-1} \Delta_{v} x_{k}-\sum_{k=1}^{n-1} \lambda_{k} \Delta_{v} x_{k}\right] \\
& =\frac{1}{\lambda_{n}}\left[\lambda_{n-1} \Delta_{v} x_{n}-\sum_{k=0}^{n-1}\left(\lambda_{k}-\lambda_{k-1}\right) \Delta_{v} x_{k}\right] \\
& =\frac{\lambda_{n-1}}{\lambda_{n}}\left[\Delta_{v} x_{n}-\tilde{\Lambda}_{n-1}(x)\right] \\
& =\frac{\lambda_{n-1}}{\lambda_{n}}\left[S_{n}(x)+\tilde{\Lambda}_{n}(x)-\tilde{\Lambda}_{n-1}(x)\right]
\end{aligned}
$$

Hence, we have for every $x \in w$ that

$$
S_{n}(x)=\frac{\lambda_{n-1}}{\lambda_{n}-\lambda_{n-1}}\left[\tilde{\Lambda}_{n}(x)-\tilde{\Lambda}_{n-1}(x)\right] \quad(n \in \mathbb{N}) .
$$

Theorem 4.4. The inclusion $\ell_{\infty} \subset \ell_{\infty}^{\lambda}\left(\Delta_{v}\right)$ strictly holds if and only if

$$
\lim _{n \rightarrow \infty} \inf \frac{\lambda_{n+1}}{\lambda_{n}}=1
$$

Proof. If we suppose that the inclusion $\ell_{\infty} \subset \ell_{\infty}^{\lambda}\left(\Delta_{v}\right)$ is strict, then Lemma $4.2 \mathrm{im}-$ plies the existence of a sequence $x \in \ell_{\infty}^{\lambda}\left(\Delta_{v}\right)$ such that $S(x)=(S(x))_{n=0}^{\infty} \notin \ell_{\infty}$. Since $x \in \ell_{\infty}^{\lambda}\left(\Delta_{v}\right)$, we have $\tilde{\Lambda}(x)=(\tilde{\Lambda}(x))_{n=0}^{\infty} \in \ell_{\infty}$ and hence $\left(\tilde{\Lambda}_{n}(x)-\tilde{\Lambda}_{n-1}(x)\right)_{n=0}^{\infty} \in$ $\ell_{\infty}$. Therefore, we deduce from (4.1) that $\left(\left(\lambda_{n-1} / \lambda_{n}-\lambda_{n-1}\right)\right)_{n=0}^{\infty} \notin \ell_{\infty}$ and hence $\left(\left(\lambda_{n} / \lambda_{n}-\lambda_{n-1}\right)\right)_{n=0}^{\infty} \notin \ell_{\infty}$. This leads us with part (a) of Lemma 4.5 [see 10] to the consequence that $\lim _{n \rightarrow \infty} \inf \lambda_{n+1} / \lambda_{n}=1$. To prove the sufficiency, suppose that $\lim _{n \rightarrow \infty} \inf \lambda_{n+1} / \lambda_{n}=1$. Then, we have by part (a) of Lemma 4.5 [see 10] that $\left(\left(\lambda_{n} / \lambda_{n}-\lambda_{n-1}\right)\right)_{n=0}^{\infty} \notin \ell_{\infty}$. Let us now define the sequence $x=\left(x_{k}\right)$ by $x_{k}=$ 
$v_{k}^{-1} \sum_{i=1}^{k}(-1)^{i} \lambda_{i} /\left(\lambda_{i}-\lambda_{i-1}\right)$ for all $k$. Then, we have for every $n \in \mathbb{N}$ that

$$
\left|\tilde{\Lambda}_{n}(x)\right|=\frac{1}{\lambda_{n}}\left|\sum_{k=0}^{n}(-1)^{k} \lambda_{k}\right| \leq \frac{1}{\lambda_{n}} \sum_{k=0}^{n}\left(\lambda_{k}-\lambda_{k-1}\right)=1
$$

which shows that $\tilde{\Lambda}_{n}(x) \in \ell_{\infty}$. Thus, the sequence $x$ is in the $\ell_{\infty}^{\lambda}\left(\Delta_{v}\right)$ but not in $\ell_{\infty}$. Therefore, by combining this with the fact that the inclusion $\ell_{\infty} \subset \ell_{\infty}^{\lambda}\left(\Delta_{v}\right)$ always holds by Lemma 4.2, we obtain that this inclusion is strict.

Corollary 4.1. The equality $\ell_{\infty}^{\lambda}\left(\Delta_{v}\right)=\ell_{\infty}$ holds if and only if $\lim _{n \rightarrow \infty} \inf \lambda_{n+1} / \lambda_{n}>1$.

Proof. The necessity is immediate by Theorem 4.4. For, if the equalities hold then the inclusions in Theorem 4.4, cannot be strict and hence $\lim _{n \rightarrow \infty} \inf \lambda_{n+1} / \lambda_{n} \neq 1$ which implies that $\lim _{n \rightarrow \infty} \inf \lambda_{n+1} / \lambda_{n}>1$. Conversely, suppose that $\lim _{n \rightarrow \infty} \inf \lambda_{n+1} / \lambda_{n}>1$. Then, it follows by part (b) of Lemma 4.5 [see 7] that $\left(\lambda_{n} /\left(\lambda_{n}-\lambda_{n-1}\right)\right)_{n=0}^{\infty}$ and hence $\left(\lambda_{n} /\left(\lambda_{n}-\lambda_{n-1}\right)\right)_{n=0}^{\infty} \in \ell_{\infty}$. Now, let $x \in \ell_{\infty}^{\lambda}\left(\Delta_{v}\right)$ be given. Then we have $\tilde{\Lambda}(x)=(\tilde{\Lambda}(x))_{n=0}^{\infty} \in \ell_{\infty}$ and hence $\left(\tilde{\Lambda}_{n}(x)-\tilde{\Lambda}_{n-1}(x)\right)_{n=0}^{\infty} \in \ell_{\infty}$. Thus we obtain by (4.1) that $\left(S_{n}(x)\right)_{n=0}^{\infty} \in \ell_{\infty}$. This shows that $S(x) \in \ell_{\infty}$ for every $x \in \ell_{\infty}^{\lambda}\left(\Delta_{v}\right)$. Consequently, we deduce by Lemma 4.2 that the equality $\ell_{\infty}^{\lambda}\left(\Delta_{v}\right)=\ell_{\infty}$ holds.

\section{REFERENCES}

[1] B. Altay, F. Başar, Some Euler sequence spaces of non-absolute type, Ukrainian Math. J. 57(1) (2005), 1-7.

[2] C. Aydın, F. Başar, On the new sequence spaces which include the spaces $c_{0}$ and $c$, Hokkaido Math. J. 33(2) (2004), 383-398.

[3] C. Bessage and A. Pelczynski, Selected topics in infinite-dimensional topology, Warszawa, 1975.

[4] B. Choudhary, S. Nanda, Functional Analysis with Applications, John Wiley \& Sons Inc. New Delhi, 1989.

[5] R. G. Cooke, Infinite matrices and sequence spaces, London: Macmilan and Co. 1950.

[6] R. Çolak, On some generalized sequence spaces, Commun. Fac. Sci. Univ. Ank. Series 38 (1989), $35-46$.

[7] A. H. Ganie, N. A. Sheikh, On some new sequence spaces of non-absolute type and matrix transformations, Journal of Egyptian Math. Society 21 (2013), 108-114.

[8] D. J. H. Garling, On topological sequence spaces, Proc. Cambridge Phil. Soc. 63 (1967), 963-981.

[9] I. J. Maddox, Elements of Functional Analysis, 2nd ed., The University Press, Cambridge, 1988.

[10] M. Mursaleen, A. K. Noman, On the spaces of $\lambda$-convergent and bounded sequences, Thai J. Math. (2) (2010) 311-329.

[11] M. Şengönül, F. Başar, Some new Cesaro sequence spaces of non-absolute type which include the spaces $c_{0}$ and $c$, Soochow J. Math. 31(1) (2005) 107-119.

[12] A. Wilansky, Summability Through Functional Analysis, in: North-Holland Mathematics Studies, Elsevier Science Publishers, Amsterdam, New York, Oxford, 1984. 
${ }^{1}$ Department of Mathematics,

Faculty of Science,

FirAT UNIVERSITY

E-mail address: sinanercan45@gmail.com

${ }^{2}$ Department of Mathematics,

FACUlty of SCience,

FirAT UNIVERSITY

E-mail address: cigdemas78@hotmail.com 